KEK-TH-935

hep-th/0402028

\title{
Effective Potential in PP-Wave Geometry
}

\author{
Kunihito Uzawa ${ }^{\dagger a}$ and Kentaroh Yoshida*b \\ ${ }^{\dagger}$ Graduate School of Human and Environmental Studies, \\ Kyoto University, Kyoto 606-8501, Japan. \\ * Theory Devision, High Energy Accelerator Research Organization (KEK), \\ Tsukuba, Ibaraki 305-0801, Japan. \\ E-mail: ${ }^{a}$ uzawa@phys.h.kyoto-u.ac.jp \\ ${ }^{b}$ kyoshida@post.kek.jp
}

\begin{abstract}
We calculate effective potentials in scalar field theories on the maximally supersymmetric pp-wave background in ten dimensions. For this purpose we have to work in the light-cone formulation, and hence we introduce two methods to compute them in the light-cone frame. One is to use the Yan's formula for evaluating one-loop correction terms. The other is to introduce a cut-off for the light-cone momentum. These methods are also confirmed in the case of Minkowski spacetime.
\end{abstract}

Keywords: effective potential, light-front, pp-wave. 


\section{Introduction}

In the past years, pp-wave backgrounds are very focused upon [1.2]. String theories on pp-waves are exactly solvable [3,4] and play an important role in studies of AdS/CFT correspondence [5]. It is also an interesting problem to apply plane-wave geometries for cosmological models. In the recent progress, Penrose limits were applied for a variety of the space-time geometries, such as $\mathrm{AdS}_{\mathrm{p}} \times \mathrm{S}^{\mathrm{q}}$ [6, 7], AdS black holes [8, 9, 10, 11, 12], and various brane configurations (for example, 6, 7, 13, 14, 15]). More recently, the plane-wave type Friedmann-Robertson-Walker (FRW) solution was proposed in [16]. This model is not static while it is exactly solvable as

a feature of plane-wave backgrounds. Another type of time-dependent plane-wave background was also found [17] and the string theory on this background was studied [18]. In addition, an example of this type of background in eleven dimensions with extra supersymmetries was proposed in [19] and the M-theory on this background was studied in [20]. Motivated by these recent attempts, we are interested in constructing other cosmological models connected to plane-wave geometry. When we intend to construct such models, it would be a first step to consider scalar field theories on pp-wave backgrounds. So far the scalar field theories on pp-waves are discussed in [8, 10, 11,21] and scalar propagator was calculated [22. However, quantum aspects of field theories on pp-waves have not been revealed yet well.

In this paper, we will calculate effective potentials in scalar field theories on the tendimensional maximally supersymmetric pp-wave background [2]. We need the methods to calculate effective potentials in the light-front formulation. However, such methods have some difficulties and so have been discussed still now (for example, see [23]). Thus, we will discuss two methods to calculate effective potentials in the light-front formulation as a setup. One is to use the Yan's formula [24, and the other is to introduce the cut-off for the light-cone momentum. By using these methods, we will calculate effective potentials in scalar field theories in four-dimensional Minkowski spacetime in order to confirm that our methods lead to the well-known results derived by the dimensional regularization and minimal subtraction scheme. These methods can be used to calculate the effective potential in the case of pp-wave backgrounds. The resulting effective potential is similar to that in the two-dimensional Minkowski spacetime up to the numerical coefficient.

This paper is organized as follows: In section 2, we present two methods to calculate effective potentials in the light-cone frame. By using these methods effective potentials in scalar field theories in four-dimensional flat space in order to confirm our methods. In section 
3, we calculate effective potentials in scalar field theories in the ten-dimensional maximally supersymmetric pp-wave background. Section 4 is devoted to a conclusion and discussions. In appendix, we will summarize several useful formulae for the calculations in this paper.

\section{Calculi of Effective Potentials in Light-Front Formulation}

In this section we will discuss calculi of effective potentials in the light-cone formulation in the case of four-dimensional Minkowski spacetime, though our purpose in this paper is to compute them in the pp-wave case. It is inevitable in the pp-wave case to work in the light-cone formulation, but the computation of effective potentials in the light-cone frame contains some technical subtleties. Hence, it would be surely available to introduce carefully the computation method.

To begin with, we will briefly review the zeta-function regularization, which is a standard method to calculate effective potentials. Then we will introduce two methods to calculate effective potentials in the light-cone formulation.

\subsection{Standard Calculus of Effective Potentials}

Let us consider the effective potential in a $d$-dimensional scalar field theory, by using the zetafunction regularization method. The action and partition function are, respectively, given by

$$
I[\phi]=\int d^{d} x\left\{-\frac{1}{2} g^{\mu \nu} \partial_{\mu} \phi \partial_{\nu} \phi-V(\phi)\right\}, \quad Z=\int \mathcal{D} \phi \mathrm{e}^{i[\phi]},
$$

where $V(\phi)$ is a given potential. When we expand the field variable $\phi$ around the constant classical background $\phi_{\mathrm{c}}$ as $\phi=\phi_{\mathrm{c}}+\delta \phi$, the effective action $\Gamma\left[\phi_{\mathrm{c}}\right]$ is represented by

$$
\begin{aligned}
i \Gamma\left[\phi_{\mathrm{c}}\right] \equiv \ln Z & =i \int d^{d} x V\left(\phi_{\mathrm{c}}\right)+\ln \left\{\int \mathcal{D} \delta \phi \exp \left(i I_{\mathrm{q}}[\delta \phi]\right)\right\}, \\
I_{\mathrm{q}}[\delta \phi] & =-\frac{1}{2} \int d^{d} x \delta \phi\left(\square_{(d)}+V_{\mathrm{c}}^{\prime \prime}\right) \delta \phi
\end{aligned}
$$

where we defined $\square_{(d)} \equiv-g^{\mu \nu} \partial_{\mu} \partial_{\nu}$ and $V_{\mathrm{c}}^{\prime \prime} \equiv d^{2} V /\left.d \phi^{2}\right|_{\phi=\phi_{\mathrm{c}}}$. By performing the path integration, the effective potential is obtained as

$$
\begin{aligned}
\Gamma\left[\phi_{\mathrm{c}}\right] & \equiv-\int d^{d} x V_{\mathrm{eff}}\left[\phi_{\mathrm{c}}\right]=-V_{d} \cdot V_{\mathrm{eff}}\left[\phi_{\mathrm{c}}\right], \\
V_{\mathrm{eff}}\left(\phi_{\mathrm{c}}\right) & =V\left(\phi_{\mathrm{c}}\right)-\frac{i}{2 V_{d}} \operatorname{Tr} \ln \left\{M^{-2}\left(\square_{(d)}+V_{\mathrm{c}}^{\prime \prime}\right)\right\} .
\end{aligned}
$$


Here we introduced a positive scale-parameter $M$ with a mass dimension to make an argument dimensionless. We can evaluate the trace-log term by introducing the generalized zeta-function:

$$
\zeta_{\mathrm{Min}}(s)=\int \frac{d^{d} k}{(2 \pi)^{d}}\left(-k^{2}+V_{\mathrm{c}}^{\prime \prime}\right)^{-s}
$$

and then the effective potential is expressed as

$$
V_{\mathrm{eff}}\left(\phi_{\mathrm{c}}\right)=V\left(\phi_{\mathrm{c}}\right)+\frac{i}{2}\left(\left.\frac{d \zeta_{\mathrm{Min}}(s)}{d s}\right|_{s=0}+\zeta_{\mathrm{Min}}(0) \cdot \ln M^{2}\right)
$$

The evaluation of the generalized zeta-function in the usual formulation is an easy task. As an example, the effective potential in four-dimensional Minkowski spacetime is given by

$$
V_{\mathrm{eff}}\left(\phi_{\mathrm{c}}\right)=V\left(\phi_{\mathrm{c}}\right)+\frac{1}{4(4 \pi)^{2}}\left(V_{\mathrm{c}}^{\prime \prime}\right)^{2}\left\{\ln \left(\frac{V_{\mathrm{c}}^{\prime \prime}}{M^{2}}\right)-\frac{3}{2}\right\}
$$

But, the situation is quite different in the light-cone formulation, because the evaluation of the generalized zeta-function (2.4) is different from that in flat case, and it is slightly difficult and technical. In the following subsections we will present new methods for calculating the oneloop correction term in the light-cone frame, which are based on the zeta-function regularization method. Namely, we present the method to evaluate the generalized zeta-function in the lightcone formulation. One is to use the Yan's formula [24, and another is to introduce the cut-off for the light-cone momentum. These methods will be applied to the pp-wave case.

\subsection{Calculus with Yan's Formula}

We now present a method to evaluate the generalized zeta-function in the light-cone frame. One of the subtleties is a Wick rotation in the light-cone formulation. However we can avoid a Wick rotation by using the Yan's formula. This method is a slight generalization of the recent impressive work of Heinzl [23].

Let us consider the case of four-dimensional Minkowski spacetime with the light-cone coordinates $x^{ \pm}=\left(x^{0} \pm x^{3}\right) / \sqrt{2}$. Now the generalized zeta function is written as

$$
\zeta_{\mathrm{Y} 4}(s)=\int_{-\infty}^{\infty} \frac{d k_{+}}{2 \pi} \int_{-\infty}^{\infty} \frac{d k_{-}}{2 \pi} \int_{-\infty}^{\infty} \frac{d^{2} k}{(2 \pi)^{2}}\left(-2 k_{+} k_{-}+k^{2}+V_{\mathrm{c}}^{\prime \prime}\right)^{-s} .
$$

The integration for the transverse momenta leads to

$$
\zeta_{\mathrm{Y} 4}(s)=\frac{\pi}{(2 \pi)^{4}} \frac{\Gamma(s-1)}{\Gamma(s)} \int_{-\infty}^{\infty} d k_{-} \int_{-\infty}^{\infty} d k_{+}(-2)^{-s+1}\left(k_{+} k_{-}-\frac{V_{\mathrm{c}}^{\prime \prime}}{2}\right)^{-s+1}
$$


The integral for $k_{+}$needs a careful treatment of single pole $k_{+}=V_{\mathrm{c}}^{\prime \prime} / 2 k_{-}$. We shift the pole by $\epsilon$ and use the Yan's formula,

$$
\int_{-\infty}^{\infty} d k_{+}\left(k_{+} k_{-}-m^{2}+i \epsilon\right)^{-s}=2 \pi i \delta\left(k_{-}\right) \frac{(-1)^{s}}{s-1}\left(m^{2}\right)^{-s+1}
$$

and then Eq. (2.7) is rewritten as

$$
\zeta_{\mathrm{Y} 4}(s)=i \frac{1}{(4 \pi)^{2}} \frac{\Gamma(s-2)}{\Gamma(s)}\left(V_{\mathrm{c}}^{\prime \prime}\right)^{-s+2} .
$$

Note that the pole of the integrand in the 1.h.s of Eq. (2.9) is at infinity when $k_{-}=0$, and this fact leads to $\delta\left(k_{-}\right)$.

As a matter of course, the final expression (2.10) calculated in the light-cone frame leads to the standard result. It should be noted that a Wick rotation is not needed as noted in [24]. In the light-cone formulation the notion of Wick rotation is subtle since the light-cone time plays a main role instead of the usual time-coordinate. But such a subtlety can be avoided by using the Yan's formula.

We can apply the method introduced here to the pp-wave case. In the next subsection we will discuss another method to calculate effective potentials in the light-cone frame.

\subsection{Calculus with the Light-Cone Momentum Cut-Off}

We shall present another calculus of effective potentials in the light-cone frame. Here we do not use the Yan's formula but introduce a cut-off for the light-cone momentum.

To begin with, we rewrite the one-loop correction term as

$$
\operatorname{Tr} \ln \left\{\square_{(\mathrm{LC})}+V_{\mathrm{c}}^{\prime \prime}\right\}=\int \frac{d k_{+} d k_{-}}{(2 \pi)^{2}} \int \frac{d^{2} k}{(2 \pi)^{2}} \ln \left(-2 k_{+} k_{-}+k^{2}+V_{\mathrm{c}}^{\prime \prime}\right) .
$$

Then, by following the work of Heinzl [23, let us introduce a cut-off for the light-cone momentum $k_{-}$as

$$
V_{\mathrm{c}}^{\prime \prime} / \Lambda \leq\left|k_{-}\right| \leq \Lambda
$$

while the standard cut-off is given by $m^{2} / \Lambda \leq\left|k_{-}\right| \leq \Lambda$. The one-loop correction term (2.11) 
can be rewritten as

$$
\begin{aligned}
\operatorname{Tr} \ln \left\{\square_{(\mathrm{LC})}+V_{\mathrm{c}}^{\prime \prime}\right\}= & \int_{-\infty}^{\infty} \frac{d k_{+}}{2 \pi} \int_{-\infty}^{\infty} \frac{d^{2} k}{(2 \pi)^{2}}\left\{\int_{V_{\mathrm{c}}^{\prime \prime} / \Lambda}^{\Lambda} \frac{d k_{-}}{2 \pi} \ln \left(-2 k_{+} k_{-}+k^{2}+V_{\mathrm{c}}^{\prime \prime}\right)\right. \\
& \left.+\int_{-\Lambda}^{-V_{\mathrm{c}}^{\prime \prime} / \Lambda} \frac{d k_{-}}{2 \pi} \ln \left(-2 k_{+} k_{-}+k^{2}+V_{\mathrm{c}}^{\prime \prime}\right)\right\} \\
= & \int_{-\infty}^{\infty} \frac{d k_{+}}{2 \pi} \int_{V_{\mathrm{c}}^{\prime \prime} / \Lambda}^{\Lambda} \frac{d k_{-}}{2 \pi} \int_{-\infty}^{\infty} \frac{d^{2} k}{(2 \pi)^{2}} \ln \left\{-\left(2 k_{+} k_{-}\right)^{2}+\left(k^{2}+V_{\mathrm{c}}^{\prime \prime}\right)^{2}\right\} .
\end{aligned}
$$

For this expression of one-loop correction, the generalized zeta-function is

$$
\zeta_{\mathrm{LC} 4}(s)=\int_{-\infty}^{\infty} \frac{d k_{+}}{2 \pi} \int_{V_{\mathrm{c}}^{\prime \prime} / \Lambda}^{\Lambda} \frac{d k_{-}}{2 \pi} \int_{-\infty}^{\infty} \frac{d^{2} k}{(2 \pi)^{2}}\left\{-\left(2 k_{+} k_{-}\right)^{2}+\left(k^{2}+V_{\mathrm{c}}^{\prime \prime}\right)^{2}\right\}^{-s} .
$$

By performing the analytic continuation $k_{+} \rightarrow i k_{+}^{*}$, we can carry out the integration for $k_{+}$. As the result, we obtain the following expression

$$
\begin{aligned}
\zeta_{\mathrm{LC} 4}(s) & =i \frac{\sqrt{\pi} \Gamma\left(s-\frac{1}{2}\right)}{(2 \pi)^{4} \Gamma(s)} \int_{-\infty}^{\infty} d^{2} k\left(k^{2}+V_{\mathrm{c}}^{\prime \prime}\right)^{-2 s+1} \int_{V_{\mathrm{c}}^{\prime \prime} / \Lambda}^{\Lambda} \frac{d k_{-}}{2 k_{-}} \\
& =-i \frac{\pi^{3 / 2} \Gamma\left(s-\frac{1}{2}\right)}{2(2 \pi)^{4} \Gamma(s)} \frac{\Gamma(2 s-2)\left(V_{\mathrm{c}}^{\prime \prime}\right)^{-2 s+2}}{\Gamma(2 s-1)} \ln \left(\frac{V_{\mathrm{c}}^{\prime \prime}}{\Lambda^{2}}\right),
\end{aligned}
$$

and the derivative of it is

$$
\zeta_{\mathrm{LC} 4}^{\prime}(s)=-i \frac{\pi^{3 / 2} \Gamma\left(s-\frac{1}{2}\right) \psi(s)}{2(2 \pi)^{4} \Gamma(s)} \frac{\Gamma(2 s-2)\left(V_{\mathrm{c}}^{\prime \prime}\right)^{-2 s+2}}{\Gamma(2 s-1)} \ln \left(\frac{V_{\mathrm{c}}^{\prime \prime}}{\Lambda^{2}}\right) .
$$

Here $\psi(s)$ is a polygamma function. The $s \rightarrow 0$ limits of them are, respectively,

$$
\lim _{s \rightarrow 0} \zeta_{\mathrm{LC} 4}(s)=0, \quad \lim _{s \rightarrow 0} \zeta_{\mathrm{LC} 4}^{\prime}(s)=-i \frac{1}{2(4 \pi)^{2}}\left(V_{\mathrm{c}}^{\prime \prime}\right)^{2} \ln \left(\frac{V_{\mathrm{c}}^{\prime \prime}}{\Lambda^{2}}\right),
$$

and hence the resulting effective potential is

$$
V_{\text {eff }}\left(\phi_{\mathrm{c}}\right)=V\left(\phi_{\mathrm{c}}\right)+\frac{1}{4(4 \pi)^{2}}\left(V_{\mathrm{c}}^{\prime \prime}\right)^{2} \ln \left(\frac{V_{\mathrm{c}}^{\prime \prime}}{\Lambda^{2}}\right) .
$$

Now we should notice the ambiguity of multiplicative constant of the cut-off $\Lambda$. When we rescale $\Lambda$ as $\Lambda^{2} \rightarrow \Lambda^{2} \mathrm{e}^{3 / 2} \equiv M^{2}$, the effective potential is identical with the standard result.

The method presented here can be also applicable to the pp-wave case. This kind of application will be discussed in the next section.

*The ambiguity of the sign exists, but we choose the sign which leads to the same result as in the standard calculation. 


\section{Effective Potentials on PP-Wave Background}

In this section we consider the effective potential in scalar field theories on the ten-dimensional maximally supersymmetric background 2]. This background is described by

$$
d s^{2}=-2 d x^{+} d x^{-}-\mu^{2} r^{2}\left(d x^{+}\right)^{2}+\sum_{i=1}^{8} d x_{i} d x^{i}
$$

where $r^{2}=\sum_{i=1}^{8}\left(x^{i}\right)^{2}$ and the parameter $\mu$ is related to the constant flux of Ramond-Ramond four-form

$$
F_{+1234}=F_{+5678}=4 \mu .
$$

This background is obtained from the $\mathrm{AdS}_{5} \times \mathrm{S}^{5}$ geometry [6, 7] via the Penrose limit [25, 26]

The action of a scalar field on this background is given by

$$
\begin{aligned}
I_{\mathrm{S}} & =\int d^{10} x \sqrt{-g}\left[-\frac{1}{2} g^{M N} \partial_{M} \phi \partial_{N} \phi-V(\phi)\right] \\
& =\int d^{10} x\left[\partial_{+} \phi \partial_{-} \phi-\frac{1}{2} \mu^{2} r^{2} \partial_{-} \phi \partial_{-} \phi-\frac{1}{2} \partial_{i} \phi \partial_{i} \phi-V(\phi)\right] .
\end{aligned}
$$

The effective potential for this system is written as

$$
V_{\text {eff }}\left(\phi_{\mathrm{c}}\right)=V\left(\phi_{\mathrm{c}}\right)-\frac{i}{2 V_{10}} \operatorname{Tr} \ln \left(\square_{(\mathrm{pp})}+V_{\mathrm{c}}^{\prime \prime}\right),
$$

by using the Laplacian in the pp-wave background $\square_{(\mathrm{pp})}$, which is defined as

$$
\square_{(\mathrm{pp})}=2 \partial_{-} \partial_{+}-\nabla_{(8)}^{2}-\mu^{2} r^{2} \partial_{-}^{2},
$$

where $\nabla_{(8)}^{2}$ is the one in the transverse eight-dimensional flat-space. All we have to do for deriving the effective potential is to evaluate the trace of the Laplacian. It can be done by the use of the harmonic-oscillator techniques as discussed in [22], and then the one-loop correction term is given by

$$
-\frac{i}{2 V_{10}} \operatorname{Tr} \ln \left(\square_{(\mathrm{pp})}+V_{\mathrm{c}}^{\prime \prime}\right)=-\frac{i}{2 V_{8}} \int_{-\infty}^{+\infty} \frac{d k_{+}}{2 \pi} \int_{-\infty}^{\infty} \frac{d k_{-}}{2 \pi} \sum_{\left\{n_{i}\right\}} \ln \left(-2 k_{+} k_{-}+\left|k_{-}\right| E_{n}+V_{\mathrm{c}}^{\prime \prime}\right),
$$

where $E_{n}$ is defined as

$$
E_{n} \equiv|\mu| \sum_{i=1}^{8}\left(n_{i}+\frac{1}{2}\right)=|\mu|(n+4), \quad n \equiv \sum_{i=1}^{8} n_{i} .
$$

We will consider the $\mu>0$ case below. Notably, the traces of the discrete spectra do not lead to the volume factor in contrast with continuous spectra, while the transverse eight-dimensional 
space is not compact (i.e., $V_{8}$ is infinite). This situation originates from the fact that the pp-wave background is not compactified though it has discrete spectra because of the $(+,+)$-component of the metric. Here we will keep the factor $V_{8}$ and return to this problem later.

We will evaluate the one-loop correction term with two methods introduced in the previous section.

\subsection{Computation with Yan's Formula}

In this subsection we will calculate the effective potential with the Yan's formula in scalar field theories on the pp-wave background.

Let us evaluate the generalized zeta-function:

$$
\begin{aligned}
\zeta_{\text {Yan }}(s)= & \frac{1}{V_{8}} \sum_{n=0}^{\infty}{ }_{n+7} C_{7} \int_{-\infty}^{\infty} \frac{d k_{+}}{2 \pi}\left[\int_{0}^{\infty} \frac{d k_{-}}{2 \pi}\left(-2 k_{+} k_{-}+k_{-} E_{n}+V_{\mathrm{c}}^{\prime \prime}\right)^{-s}\right. \\
& \left.+\int_{-\infty}^{0} \frac{d k_{-}}{2 \pi}\left(-2 k_{+} k_{-}-k_{-} E_{n}+V_{\mathrm{c}}^{\prime \prime}\right)^{-s}\right],
\end{aligned}
$$

where the combinational factor ${ }_{n+7} C_{7}$ denotes the degeneracy of the sum of $n_{i}$. We can perform the integrals for $k_{+}$and $k_{-}$by using the Yan's formula (2.9). As the result, we obtain the following expression:

$$
\begin{aligned}
\zeta_{\text {Yan }}(s)= & \frac{1}{2(2 \pi)^{2} V_{8}} \sum_{n=0}^{\infty}{ }_{n+7} C_{7} \frac{1}{s-1} E_{n}^{-s+1}\left\{\int_{0}^{\infty} d k_{-} 2 \pi i \delta\left(k_{-}\right)\left(k_{-}+\frac{V_{\mathrm{c}}^{\prime \prime}}{E_{n}}\right)^{-s+1}\right. \\
& \left.+\int_{0}^{\infty} d k_{-} 2 \pi i \delta\left(-k_{-}\right)\left(k_{-}+\frac{V_{\mathrm{c}}^{\prime \prime}}{E_{n}}\right)^{-s+1}\right\} \\
= & i \frac{1}{4 \pi V_{8}} \sum_{n=0}^{\infty}{ }_{n+7} C_{7} \frac{1}{s-1}\left(V_{\mathrm{c}}^{\prime \prime}\right)^{-s+1} .
\end{aligned}
$$

Using Eqs . (A.4) and (A.6), the expression (3.9) and its derivative can be written as:

$\zeta_{\text {Yan }}(s)=i \frac{b+1}{4 \pi(s-1) V_{8}}\left(V_{\mathrm{c}}^{\prime \prime}\right)^{-s+1}, \quad \zeta_{\text {Yan }}^{\prime}(s)=-i \frac{b+1}{4 \pi(s-1) V_{8}}\left(V_{\mathrm{c}}^{\prime \prime}\right)^{-s+1}\left(\frac{1}{s-1}+\ln V_{\mathrm{c}}^{\prime \prime}\right)$,

where $b$ is a finite constant defined by

$$
b+1=\frac{1}{7 !}\left\{\zeta_{\mathrm{R}}(-7)-14 \zeta_{\mathrm{R}}(-5)+49 \zeta_{\mathrm{R}}(-3)-36 \zeta_{\mathrm{R}}(-1)\right\}=\frac{2497}{3628800} .
$$

Here we used the Riemann's zeta function $\zeta_{\mathrm{R}}(s)$. From the above results, we obtain the formulae:

$$
\lim _{s \rightarrow 0} \zeta_{\text {Yan }}(s)=-i \frac{b+1}{4 \pi V_{8}} V_{\mathrm{c}}^{\prime \prime}, \quad \lim _{s \rightarrow 0} \zeta_{\text {Yan }}^{\prime}(s)=i \frac{b+1}{4 \pi V_{8}} V_{\mathrm{c}}^{\prime \prime}\left(\ln V_{\mathrm{c}}^{\prime \prime}-1\right) .
$$


Finally the effective potential is given by

$$
V_{\text {eff }}\left(\phi_{\mathrm{c}}\right)=V\left(\phi_{\mathrm{c}}\right)-\frac{b+1}{8 \pi V_{8}} V_{\mathrm{c}}^{\prime \prime}\left\{\ln \left(\frac{V_{\mathrm{c}}^{\prime \prime}}{M^{2}}\right)-1\right\} .
$$

It should be remarked that the resulting effective potential agrees with that in the twodimensional Minkowski spacetime up to the coefficient of the one-loop correction term. Notably, it is also independent of the parameter $\mu$. Mathematically, we can see this fact by noting that the effect of $\mu$ (i.e., $E_{n}$ ) can be absorbed by shifting the light-cone momentum $k_{+}$. According to this result of the mathematical manipulation, the physical interpretation is as follows: The vacuum energies produced by the quantum fluctuations flow from transverse space to the $k_{+^{-}}$ direction due to the flux equipped with the pp-wave geometry. In fact, we can see this fact from the classical pp-wave background. The non-trivial Einstein equation for the pp-wave geometry under our consideration is $R_{++} \sim \mu^{2} \sim F_{+1234}^{2}=F_{+5678}^{2}$, and hence the existence of flux leads to non-vanishing curvature only in the $x^{+}$-direction. As the result of the energy flow, the effect of the pp-wave background would be realized only as the numerical coefficients, and thus the vacuum energy may not explicitly depend on the parameter $\mu$.

In the next subsection, we will rederive this result by using another method.

\subsection{Computation with the Light-Cone Momentum Cut-Off}

Here we will calculate the effective potential by introducing the cut-off for the light-cone momentum in the pp-wave case.

We begin with the evaluation of the one-loop correction term, which is rewritten as

$$
\operatorname{Tr} \ln \left\{\square_{(\mathrm{pp})}+V_{\mathrm{c}}^{\prime \prime}\right\}=\frac{1}{V_{8}} \int_{-\infty}^{+\infty} \frac{d k_{+}}{2 \pi} \int_{V_{\mathrm{c}}^{\prime \prime} / \Lambda}^{\Lambda} \frac{d k_{-}}{2 \pi} \sum_{\left\{n_{i}\right\}} \ln \left\{-\left(2 k_{+} k_{-}\right)^{2}+\left(k_{-} E_{n}+V_{\mathrm{c}}^{\prime \prime}\right)^{2}\right\} .
$$

Here we define the generalized zeta-function by

$$
\zeta_{\mathrm{pp}}(s)=\frac{1}{V_{8}} \sum_{n=0}^{\infty}{ }_{n+7} C_{7} \int_{V_{\mathrm{c}}^{\prime \prime} / \Lambda}^{\Lambda} \frac{d k_{-}}{2 \pi} \int_{-\infty}^{\infty} \frac{d k_{+}}{2 \pi}\left\{-4 k_{+}^{2} k_{-}^{2}+\left(k_{-} E_{n}+V_{\mathrm{c}}^{\prime \prime}\right)^{2}\right\}^{-s} .
$$

Performing the integral for $k_{+}$after the analytic continuation $k_{+} \rightarrow i k_{+}$, Eq. (3.15) is rewritten as

$$
\zeta_{\mathrm{pp}}(s)=i \frac{1}{V_{8}} \sqrt{\pi} \frac{\Gamma\left(s-\frac{1}{2}\right)}{2(2 \pi)^{2} \Gamma(s)} \sum_{n=0}^{\infty}{ }_{n+7} C_{7} E_{n}^{-2 s+1} \int_{V_{\mathrm{c}}^{\prime \prime} / \Lambda}^{\Lambda} \frac{d k_{-}}{k_{-}}\left(k_{-}+\frac{V_{\mathrm{c}}^{\prime \prime}}{E_{n}}\right)^{-2 s+1}
$$


Then the generalized zeta-function is explicitly written as

$$
\begin{aligned}
\zeta_{\mathrm{pp}}(s)=\frac{i}{V_{8}} & \sqrt{\pi} \frac{\Gamma\left(s-\frac{1}{2}\right)}{2(2 \pi)^{2} \Gamma(s)} \sum_{n=0}^{\infty}{ }_{n+7} C_{7} E_{n}^{-2 s+1} \frac{1}{2 s-1} \\
& \times\left\{{ }_{2} F_{1}\left(2 s-1,2 s-1,2 s,-\frac{\Lambda}{E_{n}}\right)\left(\frac{V_{\mathrm{c}}^{\prime \prime}}{\Lambda}\right)^{-2 s+1}\right. \\
& \left.-{ }_{2} F_{1}\left(2 s-1,2 s-1,2 s,-\frac{V_{\mathrm{c}}^{\prime \prime}}{\Lambda E_{n}}\right) \Lambda^{-2 s+1}\right\} .
\end{aligned}
$$

The hypergeometric function ${ }_{2} F_{1}$ can be expanded ${ }^{\dagger}$ as

$$
\begin{gathered}
{ }_{2} F_{1}\left(2 s-1,2 s-1,2 s,-\frac{\Lambda}{E_{n}}\right)=1-\frac{(2 s-1)^{2}}{2 s} \frac{\Lambda}{E_{n}}+\frac{s(2 s-1)^{2}}{2 s+1}\left(\frac{\Lambda}{E_{n}}\right)^{2} \\
-\frac{s(2 s-1)^{2}(2 s+1)}{3(2 s+2)}\left(\frac{\Lambda}{E_{n}}\right)^{3}+O\left\{\left(\frac{\Lambda}{\mu}\right)^{4}\right\}, \\
{ }_{2} F_{1}\left(2 s-1,2 s-1,2 s,-\frac{V_{\mathrm{c}}^{\prime \prime}}{\Lambda E_{n}}\right)=1-\frac{(2 s-1)^{2}}{2 s} \frac{V_{\mathrm{c}}^{\prime \prime}}{\Lambda E_{n}}+\frac{s(2 s-1)^{2}}{2 s+1}\left(\frac{V_{\mathrm{c}}^{\prime \prime}}{\Lambda E_{n}}\right)^{2} \\
-\frac{s(2 s-1)^{2}(2 s+1)}{3(2 s+2)}\left(\frac{V_{\mathrm{c}}^{\prime \prime}}{\Lambda E_{n}}\right)^{3}+O\left\{\left(\frac{V_{\mathrm{c}}^{\prime \prime}}{\Lambda \mu}\right)^{4}\right\},
\end{gathered}
$$

and the generalized zeta-function (3.17) can be rewritten as

$$
\begin{aligned}
\zeta_{\mathrm{pp}}(s) & =\frac{i \sqrt{\pi}}{V_{8}}\left[\frac{1}{2(2 \pi)^{2}} \frac{\Gamma\left(s-\frac{1}{2}\right)}{(2 s-1) \Gamma(s)} \sum_{n=0}^{\infty}{ }_{n+7} C_{7}\{\mu(n+4)\}^{-2 s+1}\left\{\left(\frac{V_{\mathrm{c}}^{\prime \prime}}{\Lambda}\right)^{-2 s+1}-\Lambda^{-2 s+1}\right\}\right. \\
& -\frac{1}{2(2 \pi)^{2}} \frac{2 s-1}{2 s} \frac{\Gamma\left(s-\frac{1}{2}\right)}{\Gamma(s)} \sum_{n=0}^{\infty}{ }_{n+7} C_{7}\{\mu(n+4)\}^{-2 s} V_{\mathrm{c}}^{\prime \prime}\left\{\left(\frac{V_{\mathrm{c}}^{\prime \prime}}{\Lambda}\right)^{-2 s}-\Lambda^{-2 s}\right\} \\
& \left.+O\left(\frac{1}{\mu^{2 s+1}}\right)\right] .
\end{aligned}
$$

Using Eqs. (3.20), (A.4) and (A.7) leads to the limits:

$$
\lim _{s \rightarrow 0} \zeta_{\mathrm{pp}}(s)=0, \quad \lim _{s \rightarrow 0} \zeta_{\mathrm{pp}}^{\prime}(s)=i \frac{b+1}{4 \pi V_{8}} V_{\mathrm{c}}^{\prime \prime} \ln \left(\frac{V_{\mathrm{c}}^{\prime \prime}}{\Lambda^{2}}\right) .
$$

Here $b$ is defined in Eq. (3.11). It should be noted that higher order terms $O(1 / \mu)$ vanish in the limit $s \rightarrow 0$.

\footnotetext{
${ }^{\dagger}$ We comment on the convergence radius of the expansion with respect to $1 / \mu$. In the large $\mu$, the hypergeometric function ${ }_{2} F_{1}$ is expanded in terms of the $\Lambda / \mu$ and $V_{\mathrm{c}}^{\prime \prime} /(\Lambda \mu)$. Then $\zeta_{\text {pp }}$ converges for the case $\Lambda<\mu$ and $V_{\mathrm{c}}^{\prime \prime}<\Lambda \mu$.
} 
Finally, the effective potential is represented by

$$
V_{\text {eff }}\left(\phi_{\mathrm{c}}\right)=V\left(\phi_{\mathrm{c}}\right)-\frac{b+1}{8 \pi V_{8}} V_{\mathrm{c}}^{\prime \prime} \ln \left(\frac{V_{\mathrm{c}}^{\prime \prime}}{\Lambda^{2}}\right) .
$$

When we define the scale parameter $M$ in Eq. (3.13) as $M^{2}=\Lambda^{2}$ e, the above expression becomes identical with the previous result derived by using the Yan's formula.

Now let us comment on the vacuum energy. We consider the quadratic potential of scalar field defined by $V(\phi)=m^{2} \phi^{2} / 2$. By inserting it into the effective potential (3.13) (or (3.22) ), we obtain

$$
V_{\mathrm{eff}}\left(\phi_{\mathrm{c}}\right)=\frac{1}{2} m^{2} \phi_{\mathrm{c}}^{2}-\frac{b+1}{8 \pi V_{8}} m^{2}\left\{2 \ln \left(\frac{m}{M}\right)-1\right\}
$$

where the second term expresses the one-loop correction and may be interpreted as the vacuum energy. It is well-known that the zero-point energy of massive fields may contribute to the cosmological constant.

From now on, we discuss the volume of eight-dimensional transverse space. It would be plausible to measure the volume with the damping length of the wave packet in the system of harmonic oscillators. That is, the volume $V_{8}$ is regarded as the moving region of harmonic oscillator (See Fig. (1). This length is estimated as $\left(\mu k_{-}\right)^{-1 / 2}$. When we assume that the cut-off scale for $k_{-}$is imposed by $V_{\mathrm{c}}^{\prime \prime} / \Lambda \leq\left|k_{-}\right| \leq \Lambda$, the effective potential for the infrared cut-off $V_{\mathrm{c}}^{\prime \prime} / \Lambda$ is

$$
V_{\mathrm{eff}}\left(\phi_{\mathrm{c}}\right) \sim V\left(\phi_{\mathrm{c}}\right)-\frac{b+1}{8 \pi}\left(\frac{\mu}{\Lambda}\right)^{4}\left(V_{\mathrm{c}}^{\prime \prime}\right)^{5}\left\{\ln \left(\frac{V_{\mathrm{c}}^{\prime \prime}}{\Lambda^{2}}\right)-1\right\} .
$$

This is just an effective potential of scalar field in ten-dimensional Minkowski spacetime. On the other hand, the effective potential for ultraviolet cut-off $\Lambda$ is given by

$$
V_{\text {eff }}\left(\phi_{\mathrm{c}}\right) \sim V\left(\phi_{\mathrm{c}}\right)-\frac{b+1}{8 \pi}(\mu \Lambda)^{4} V_{\mathrm{c}}^{\prime \prime}\left\{\ln \left(\frac{V_{\mathrm{c}}^{\prime \prime}}{\Lambda^{2}}\right)-1\right\} .
$$

This result is equivalent to that in two-dimensional Minkowski spacetime.

Finally we shall briefly comment on the limit $\mu \rightarrow 0$. We can recover the effective potential in ten-dimensional Minkowski spacetime from (3.6) by following the replacement law:

$$
\mu k_{-}\left(n_{i}+\frac{1}{2}\right) \Rightarrow k_{i}^{2}, \quad \frac{1}{V_{8}} \sum_{\left\{n_{i}\right\}} \Rightarrow \int \frac{d^{8} k}{(2 \pi)^{8}} .
$$

What does it physically mean? If we take the limit $\mu \rightarrow 0$ naively, then we cannot recover the transverse momentum. We need to take account of the energy conservation of the harmonic 


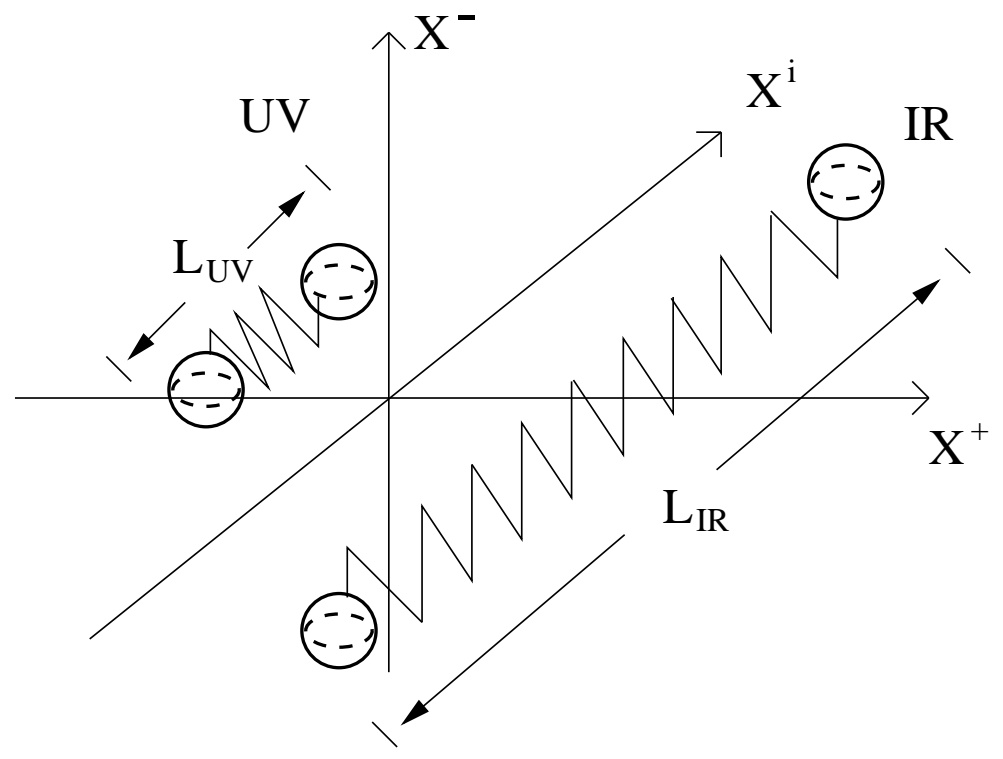

Figure 1: This figure shows the "effective" compactification for the transverse directions. The effective scale for the transverse direction is evaluated by $\left(\mu k_{-}\right)^{-1 / 2}$. Then the moving region at the scale $\Lambda$ (UV) is far shorter than that of $V_{\mathrm{c}}^{\prime \prime} / \Lambda$ (IR) when the cut-off scale $\Lambda$ is assumed to be large enough.

oscillations. The above relations mean that the energy of the harmonic oscillators should be replaced with the kinematic energy of free motion. In other words, we have to take account of the Casimir energy.

Once we suppose the above replacement law, we can easily obtain the effective potential in ten-dimensional Minkowski spacetime

$$
V_{\text {eff }}\left(\phi_{\mathrm{c}}\right)=V\left(\phi_{\mathrm{c}}\right)-\frac{1}{15 \cdot 2^{4}(4 \pi)^{5}}\left(V_{\mathrm{c}}^{\prime \prime}\right)^{5}\left\{\ln \left(\frac{V_{\mathrm{c}}^{\prime \prime}}{\Lambda^{2}}\right)-\frac{137}{60}\right\}
$$

by using

$$
\lim _{s \rightarrow 0}\left\{\frac{\Gamma(s-5)}{\Gamma(s)}\right\}=-\frac{1}{120}, \quad \frac{\Gamma(s-5)\{\psi(s-5)-\psi(s)\}}{\Gamma(s)}=\frac{1}{120}\left(-\frac{137}{60}\right)+O(s) .
$$

\section{Conclusion and Discussion}

We have developed two methods to calculate effective potentials in the light-cone frame for the application in the pp-wave case. One is to use the Yan's formula and the other is to introduce a cut-off for the light-cone momentum. These methods would be available for the study of the 
usual theories such as QED and QCD in the light-front formulation other than quantum field theories on pp-wave backgrounds.

By using our two methods, we have calculated the effective potential in a scalar field theory on the ten-dimensional maximally supersymmetric pp-wave background. The effective potential obtained here is similar to that in two-dimensional Minkowski spacetime up to the coefficient of the one-loop correction term. It would be an important subject to compute effective potentials in the canonical formulation of the discrete light-cone quantization (DLCQ) $)^{\ddagger}$ and to confirm our result in this paper, while we have utilized the path integral formulation in this paper. We will study in this direction and report in the near future [28].

There are many future problems. The pp-wave background considered here is static and the scalar field theory on this background is not realistic as a cosmological model. Thus, it is one of the most interesting problems to consider scalar field theories on time-dependent planewave backgrounds as in [16, 17, 18, 19, 20]. Also, it might be possible to apply our methods to the case of time-dependent orbifolds. Moreover, it is nice to consider scalar field theories on four-dimensional pp-wave backgrounds.

In addition, it is interesting to study effective potentials in scalar field theories on planewave backgrounds obtained from (AdS) black holes via Penrose limits [9, 10, 11, 12, In these cases we would face a harmonic oscillator with a negative mass-square, and hence the behavior of the sum of modes (i.e., one-loop corrections) would be drastically modified. It has been, however, argued in the literatures [9, 10,11] that such a negative mass square would not lead to an imaginary part in the effective potential and hence a break-down of unitarity would not be caused. It would be an attractive work to check this argument from the viewpoint of one-loop effective potential in order to promote our understanding of pp-wave geometry and Penrose limit.

\section{Acknowledgment}

We would like to thank M. Sakagami and K. Sugiyama for continuing encouragement and helpful discussion. We also thank T. Uematsu for useful comments.

\footnotetext{
${ }^{\ddagger}$ As an example of the works for such a direction in flat space, we can refer to 27 .
} 


\section{Appendix}

\section{A Useful Formulae}

In this appendix A, we shall summarize useful formulae for the computation of effective potential by using the zeta function regularization method. These are surely available for the calculation in section 3

\section{Integration Formula}

In order to compute effective potentials, we first use the following integration formulae:

$$
\begin{gathered}
\int_{-\infty}^{\infty} d^{d} x\left(x^{2}+M^{2}\right)^{-s}=\pi^{d / 2} \frac{\Gamma\left(s-\frac{d}{2}\right)}{\Gamma(s)}\left(M^{2}\right)^{-s+d / 2} \\
\int_{\Lambda_{1}}^{\Lambda_{2}} d x \frac{\left(x+M^{2}\right)^{-s}}{x}=\frac{1}{s}\left\{\Lambda_{1}^{-s}{ }_{2} F_{1}\left(s, s, s+1,-\frac{M^{2}}{\Lambda_{1}}\right)\right. \\
\left.-\Lambda_{2}^{-s}{ }_{2} F_{1}\left(s, s, s+1,-\frac{M^{2}}{\Lambda_{2}}\right)\right\}
\end{gathered}
$$

where ${ }_{2} F_{1}(\alpha, \beta, \gamma, z)$ is the Gauss's hypergeometric function:

$$
{ }_{2} F_{1}(\alpha, \beta, \gamma, z)=\frac{\Gamma(\gamma)}{\Gamma(\alpha) \Gamma(\beta)} \sum_{n=0}^{\infty} \frac{\Gamma(\alpha+n) \Gamma(\beta+n)}{\Gamma(\gamma+n)} \frac{z^{n}}{n !} .
$$

\section{Formulae of Summation}

In the calculation of effective potential in scalar field theories on a pp-wave background, we have to evaluate the following infinite sum:

$$
\sum_{n=0}^{\infty}{ }_{n+7} C_{7}(n+4)^{-s-d}
$$

The sum without the combinatorial factor can be rewritten as

$$
\sum_{n=0}^{\infty}(n+4)^{-s-d}=\zeta_{\mathrm{R}}(s+d)-1-2^{-s-d}-3^{-s-d}+4^{-s-d},
$$

by using the Riemann's zeta function $\zeta_{\mathrm{R}}(s)$ defined as

$$
\zeta_{\mathrm{R}}(s)=\sum_{n=1}^{\infty} n^{-s}
$$


On the other hand, noting that the combinatorial factor ${ }_{n+7} C_{7}$ can be represented by

$$
{ }_{n+7} C_{7}=\frac{1}{7 !}\left\{(n+4)^{7}-14(n+4)^{5}+49(n+4)^{3}-36(n+4)\right\},
$$

one can obtain the following relation:

$$
\begin{aligned}
\sum_{n=0}^{\infty}{ }_{n+7} C_{7}(n+4)^{-s-d}= & \frac{1}{7 !}\left\{\zeta_{\mathrm{R}}(s+(d-7))-14 \zeta_{\mathrm{R}}(s+(d-5))\right. \\
& \left.+49 \zeta_{\mathrm{R}}(s+(d-3))-36 \zeta_{\mathrm{R}}(s+(d-1))+4^{-s-d}\right\} .
\end{aligned}
$$

This formula is also useful in the calculation in section 3 .

\section{References}

[1] J. Kowalski-Glikman, "Vacuum States In Supersymmetric Kaluza-Klein Theory," Phys. Lett. B 134 (1984) 194.

[2] M. Blau, J. Figueroa-O'Farrill, C. Hull and G. Papadopoulos, "A new maximally supersymmetric background of IIB superstring theory," JHEP 0201 (2002) 047 arXiv:hep-th/0110242.

[3] R. R. Metsaev, "Type IIB Green-Schwarz superstring in plane wave Ramond-Ramond background," Nucl. Phys. B 625 (2002) 70 arXiv:hep-th/0112044.

[4] R. R. Metsaev and A. A. Tseytlin, "Exactly solvable model of superstring in plane wave Ramond-Ramond background," Phys. Rev. D 65 (2002) 126004 arXiv:hep-th/0202109.

[5] D. Berenstein, J. Maldacena and H. Nastase, "Strings in flat space an pp waves from $\mathcal{N}=4$ Super Yang Mills," JHEP 0204 (2002) 013 arXiv:hep-th/0202021.

[6] M. Blau, J. Figueroa-O'Farrill, C. Hull and G. Papadopoulos, "Penrose limits and maximal supersymmetry," Class. Quant. Grav. 19 (2002) L87 arXiv:hep-th/0201081.

[7] M. Blau, J. Figueroa-O'Farrill and G. Papadopoulos, "Penrose limits, supergravity and brane dynamics," Class. Quant. Grav. 19 (2002) 4753 arXiv:hep-th/0202111.

[8] D. Marolf and S. F. Ross, "Plane waves: To infinity and beyond!," Class. Quant. Grav. 19 (2002) 6289 arXiv:hep-th/0208197. 
[9] V. E. Hubeny and M. Rangamani, "No horizons in pp-waves," JHEP 0211 (2002) 021 arXiv:hep-th/0210234.

[10] D. Brecher, J. P. Gregory and P. M. Saffin, "String theory and the classical stability of plane waves," Phys. Rev. D 67 (2003) 045014 arXiv:hep-th/0210308.

[11] D. Marolf and L. A. Pando Zayas, "On the singularity structure and stability of plane waves," JHEP 0301 (2003) 076 arXiv:hep-th/0210309.

[12] M. Li, "PP-wave black holes and the matrix model," JHEP 0305 (2003) 031 arXiv:hep-th/0212345.

[13] Y. Oz and T. Sakai, "Penrose limit and six-dimensional gauge theories," Phys. Lett. B 544 (2002) 321 arXiv:hep-th/0207223.

[14] H. Fuji, K. Ito and Y. Sekino, "Penrose limit and string theories on various brane backgrounds," JHEP 0211 (2002) 005 arXiv:hep-th/0209004.

[15] K. Ito and Y. Sekino, "Penrose limit and enhancon geometry," Phys. Rev. D 67 (2003) 126005 arXiv:hep-th/0302127.

[16] G. Papadopoulos, J. G. Russo and A. A. Tseytlin, "Solvable model of strings in a time-dependent plane-wave background," Class. Quant. Grav. 20 (2003) 969 arXiv:hep-th/0211289.

[17] M. Blau and M. O’Loughlin, "Homogeneous plane waves," Nucl. Phys. B 654 (2003) 135 arXiv:hep-th/0212135.

[18] M. Blau, M. O'Loughlin, G. Papadopoulos and A. A. Tseytlin, "Solvable models of strings in homogeneous plane wave backgrounds," Nucl. Phys. B 673 (2003) 57 arXiv:hep-th/0304198.

[19] M. Blau, P. Meessen and M. O’Loughlin, "Goedel, Penrose, anti-Mach: Extra supersymmetries of time-dependent plane waves," JHEP 0309 (2003) 072 arXiv:hep-th/0306161.

[20] M. Sakaguchi and K. Yoshida, "M-theory on a time-dependent plane-wave," JHEP 0311 (2003) 030 arXiv:hep-th/0309025. 
[21] D. Berenstein and H. Nastase, "On lightcone string field theory from super Yang-Mills and holography," arXiv:hep-th/0205048.

[22] S. D. Mathur, A. Saxena and Y. K. Srivastava, "Scalar propagator in the pp-wave geometry obtained from $\mathrm{AdS}_{5} \times \mathrm{S}^{5}$," Nucl. Phys. B 640 (2002) 367 arXiv:hep-th/0205136.

[23] T. Heinzl, "The light-cone effective potential," arXiv:hep-th/0212202.

[24] T. M. Yan, "Quantum Field Theories In The Infinite Momentum Frame. 4. Scattering Matrix Of Vector And Dirac Fields And Perturbation Theory," Phys. Rev. D 7 (1973) 1780 .

[25] R. Penrose, "Any spacetime has a plane wave as a limit," Differential geometry and relativity, Reidel, Dordrecht, 1976, pp. 271-275.

[26] R. Güven, "Plane wave limits and T-duality," Phys. Lett. B 482 (2000) 255 arXiv:hep-th/0005061.

[27] T. Heinzl, "Light-cone quantization: Foundations and applications," Lect. Notes Phys. 572 (2001) 55 arXiv:hep-th/0008096 and references therein.

[28] K. Uzawa and K. Yoshida, in preperation. 